\title{
Cross-Market Infection Research on Stock Herding Behavior Based on DGC-MSV Models and Bayesian Network
}

\author{
Jing Zhang $\mathbb{i}$ and Ya-ming Zhuang $(\mathbb{D}$ \\ School of Economics and Management, Southeast University, Nanjing, Jiangsu, China \\ Correspondence should be addressed to Ya-ming Zhuang; 101010407@seu.edu.cn
}

Received 16 December 2020; Revised 30 December 2020; Accepted 12 January 2021; Published 22 January 2021

Academic Editor: M. Irfan Uddin

Copyright (c) 2021 Jing Zhang and Ya-ming Zhuang. This is an open access article distributed under the Creative Commons Attribution License, which permits unrestricted use, distribution, and reproduction in any medium, provided the original work is properly cited.

\begin{abstract}
This paper is concerned with the multivariate stochastic volatility modeling of the stock market. We investigate a DGC-t-MSV model to find the historical volatility spillovers between nine markets, including S\&P, Nasdaq, SSE, SZSE, HSI, FTSE, CAC, DAX, and Nikkei indices. We use the Bayesian network to analyze the spreading of herd behavior between nine markets. The main results are as follows: (1) the DGC-t-MSV model we considered is a useful way to estimate the parameter and fit the data well in the stock market; (2) our computational analysis shows that the S\&P and Nasdaq have higher volatility spillovers to the Shanghai and Shenzhen stock markets; (3) the results also show that there is a strong correlation between stock markets in the same region.
\end{abstract}

\section{Introduction}

Price volatility in the stock market is the result of a combination of factors in the whole economy. Through the observation of historical data, we can analyze the fluctuation over a period of time. The ARCH model [1] and stochastic volatility model [2] are two main models for studying the volatility of the time series. The SV model adds a random factor to the ARCH model, which makes the SV model better to fit the real stock fluctuations. The SV model is widely used to analyze the high-frequency financial time series [3, 4]. Some researchers make various improved models based on the SV model, including added nonlinear [5] and mean equation [6], added an interrelated time series [7], added leverage and student distribution [8,9], and added twofactor simulation [10].

The study of the investor's behavior shows that the herd behavior does not only appear in a certain market but widely exists in various stock markets [11]. The crisis will cause herd behavior in the country where the crisis originated and spread to neighboring countries. However, the diversified asset plays an important role to explain the herding behavior in non-US markets [11]. So, the herd behavior exists not only in developed countries but also in emerging markets and underdeveloped markets [12].

Research on herd behavior has focused on a single market, such as the study of herd behavior in Chinese stock markets [13]. More and more herd behavior research studies began to pay attention to the spillover between markets. The authors in [14] find the herd behavior caused by the unexpected impact from both China and US markets and have proved that the Chinese market has a weaker response to the impact from the US market. After the financial crisis in 2008, there is growing evidence that herd behavior in different markets is likely to be driven by the same information at the same time $[15,16]$. There are also studies of herd behavior with intercontinental features [17] and time-varying features [18]. Volatility spillover causes comovement between markets [19], especially in crisis [20, 21].

Bayesian networks can reflect the uncertainty relationship between different markets. The Bayesian network is widely used in analysis and diagnosis [22]. The complex network and Bayesian network are often used in the stock market to reflect the interaction between traders [23-25]. The Bayesian method is also used to predict the stock market [26] and estimate volatility parameters [27]. Malagrino et al. 
[28] use the Bayesian network to verify the impact of the global stock market index on iBOVESPA.

Existing research focuses on herd behavior in one market. But with the integration of the world economy, the spread of herd behavior is no longer limited to a certain market. It is necessary to research on cross-market effects of the stock market volatility and herd behavior. In this paper, an improved multivariate stochastic volatility model is used to analyze the historical data of S\&P, Nasdaq, SSE, SZSE, HSI, FTSE, CAC, DAX, and Nikkei indices. Then, we use the volatility spillover correlation parameters between nine markets to build a Bayesian network.

The article is organized as follows. Section 2 introduces the DGC-MSV-t model and the Bayesian network model. Section 3 analyzes the weekly data of nine representative stock indexes and establishes a Bayesian network based on the volatility spillover relationships. Section 4 concludes this paper.

\section{Stochastic Volatility Model and Bayesian Network Classifiers}

\subsection{Multivariate Stochastic Volatility Model}

\subsubsection{Stochastic Volatility Model and DGC-MSV Model.}

$$
\begin{aligned}
p_{t} & =\exp \left(\frac{q_{t}}{2}\right) \varepsilon_{t},{ }_{\varepsilon_{t}^{\sim}}^{i i d} N\left(0, \Sigma_{\varepsilon, t}\right), \\
q_{t+1} & =\mu+\psi_{1}\left(L_{t}-\mu\right)+\xi_{t}, \varepsilon_{t}^{i i d} N\left(0, \sigma^{2}\right) .
\end{aligned}
$$

In equation (1), the known parameter is the yield sequence $p_{t}$, the unknown parameter $q_{t}$ and $\psi_{\mathrm{t}}$ are unobservable variables, $p_{t}$ represents the standard logarithmic yield at time $t, \varepsilon_{t}$ indicates the logarithmic volatility, $\xi_{\mathrm{t}}$ indicates the independent disturbance of the volatility, $\xi_{\mathrm{t}}$ and $\varepsilon_{t}$ are irrelevant, $\sigma$ represents the standard error of the volatility disturbance, and $\psi_{1}$ is a continuous parameter that reflects the impact of current volatility on future.

Based on the GC-MSV model and the DC-MSV model proposed by $\mathrm{Yu}$ and Meyer [29], we build the multivariate stochastic volatility model with dynamic correlation, Granger causality, and $t$-distribution.

$$
\begin{aligned}
p_{t} & =\exp \left(\frac{q_{t}}{2}\right) \varepsilon_{t}, \varepsilon_{t}^{\text {iid }} \sim \underset{\sim}{\sim} T\left(0, \Sigma_{\varepsilon, t}, o\right), \sum_{\varepsilon, t}=\left(\begin{array}{cc}
1 & \rho_{t} \\
\rho_{t} & 1
\end{array}\right), \\
q_{t+1} & =\mu+\psi\left(p_{t}-\mu\right)+\xi_{t}, \varepsilon_{t}^{i i d} N\left(0, \operatorname{diag}\left(\sigma_{\xi_{a}}^{2}, \sigma_{\xi_{c}}^{2}\right)\right), \\
r_{t+1} & =v_{0}+v_{a c}\left(r_{t}-v_{0}\right)+\sigma_{\rho} o_{t}, o_{t}^{\sim i i d} N(0,1), \rho_{t}=\frac{\exp \left(r_{t}\right)-1}{\exp \left(r_{t}\right)+1} .
\end{aligned}
$$

Equation (2) has two sets of time series. Taking the SSE index and the S\&P index as examples, $p_{c}$ represents the yield of the SSE index and $p_{a}$ represents the yield of the S\&P index. $\psi=\left(\begin{array}{ll}\psi_{c c} & \psi_{c a} \\ \psi_{a c} & \psi_{a a}\end{array}\right)$, where $\psi_{a c}$ represents the volatility spillover from the SSE index to the S\&P index, $\psi_{c a}$ is the opposite, and $\psi_{a a}$ and $\psi_{c c}$ represent the autocorrelation of the SSE and S\&P index. $\rho_{t}$ represents the dynamic correlation [29]. $o$ reflects the degree of freedom.

In equations (3)-(6), we describe the design procedure of the DGC-MSV model. With a given $q_{t+1}, \mu$ and $\psi$ parameters obey $\left(\mu+\psi\left(q_{t-1}-\mu\right), \operatorname{diag}\left(\sigma_{\xi_{a}}^{2}, \sigma_{\xi_{c}}^{2}\right)\right)$ distribution as follows:

$$
\left(\frac{\theta_{t}}{\mu}\right), \psi, p_{t}^{\sim} N\left(\mu+\psi\left(q_{t}-\mu\right), \operatorname{diag}\left(\sigma_{\xi_{a}}^{2}, \sigma_{\xi_{c}}^{2}\right)\right), \quad t=1,2, \ldots, n .
$$

The density $t$ is the degree of freedom $o$ as follows:

$$
f(t ; o)=\frac{\Gamma((o+1) / 2)}{\Gamma(o / 2)} \sqrt{\frac{1}{o \pi}}\left(1+\frac{t^{2}}{o}\right)^{(-(o+1) / 2)} .
$$

Therefore, the distribution density of $f\left(p_{t} / q_{t}\right)$ is as follows:

$$
f\left(\frac{p_{t}}{q_{t}}\right)=\exp \left(-\frac{q_{t}}{2}\right) \frac{\Gamma((o+1) / 2)}{\Gamma(o / 2)} \sqrt{\frac{1}{o \pi}}\left(1+\frac{q_{t}^{2} \exp \left(-\varepsilon_{t}\right)}{o}\right)^{(-(o+1) / 2)} .
$$

That is to say, $\left(p_{t} / \varepsilon_{t}\right)$ also obeys the $t$-distribution, i.e., $\left(p_{t} /\left(\varepsilon_{t}^{\sim} t\left(\exp \left(\varepsilon_{t}\right), o\right)\right)\right)$. The likelihood function $L$ can be expressed as follows:

$$
\begin{aligned}
L\left(\mu, \psi, o, \varepsilon_{0: n}, \operatorname{diag}\left(\sigma_{\xi_{a}}^{2}, \sigma_{\xi_{c}}^{2}\right)\right) & =\prod_{t=1}^{n} f\left(\frac{p_{t}}{\varepsilon_{t}}\right)=\prod_{t=1}^{n} \sqrt{\frac{1}{o \pi}} \frac{\Gamma((o+1) / 2)}{\Gamma(o / 2)} \exp \left(-\frac{q_{t}}{2}\right) \cdot\left[1+\frac{1}{o} p_{t}^{2} \exp \left(-\varepsilon_{t}\right)\right]^{(-(o+1) / 2)} \\
& =\frac{1}{(o \pi)^{n / 2}}\left(\frac{\Gamma((o+1) / 2)}{\Gamma(o / 2)}\right)^{n} \exp \left(-\frac{1}{2} \sum_{t=1}^{n} \varepsilon_{t}\right) \cdot \prod_{t=1}^{n}\left(1+\frac{1}{o} \exp (-\psi)_{t}\right)^{(-(o+1) / 2)}
\end{aligned}
$$


2.1.2. Bayesian Estimation. The DGC-MSV model is made by a prior distribution of unknown parameters. There are 9 unknown parameters, including $q_{1}, q_{2}, \xi_{1}, \xi_{2}, \psi_{1}, \psi_{2}, \sigma_{12}, \sigma_{21}$, and $o$. Vector $\kappa$ contains all unknown parameters and potential $\log$ volatility, i.e., $\kappa=\left(a, q_{1}, \ldots, q_{T}\right)$.

$$
h(a) h\left(q_{0}\right) \prod_{t=1}^{T} h\left(\frac{q_{t}}{a}\right)=h\left(p_{1}\right) h\left(p_{2}\right) h\left(\xi_{1}\right) h\left(\xi_{2}\right) h\left(\psi_{1}\right) h\left(\psi_{2}\right) h\left(\sigma_{12}\right) h\left(\sigma_{21}\right) h(o) h\left(q_{0}\right) \prod_{t-1}^{T} h\left(\frac{q_{t}}{a}\right) .
$$

In order to calculate the marginal posterior distribution of the target parameter, $h(a / p)$ needs to find the normalization constant in $(h+2 T)$-dimensional integral $h(\varepsilon) h(f / \varepsilon) \mathrm{d} \varepsilon$ as follows:

$$
p\left(\frac{a}{p}\right)=\int_{h_{1}} \ldots \int_{h_{T}} h\left(a, q_{1}, \ldots, q_{T}\right) d q_{T}, \ldots, d q_{1} .
$$

The MCMC method has proven to be the most effective way to solve the high-dimensional calculation problem [30]. Here, we use the WinBUGS software package to calculate this problem of the multivariate SV model.

2.1.3. Markov chain. The Markov chain assumes that the parameter of the random process $\left\{\mathrm{X}_{\mathrm{t}}, t \in T\right\}$ is a discrete set $I=\left\{x_{1}, x_{2}, \ldots\right\}$. We use the Markov chain as follows:

$$
P\left\{X_{0}=x_{0}, X_{1}=x_{1}, \ldots, X_{t}=x_{t}\right\}=P\left(X_{0}=x_{0}\right) \prod_{t-1}^{t} P \frac{\left(X_{i}=x_{i}\right)}{\left(X_{i-1}=x_{i-1}\right)}
$$

Therefore, the statistical properties of the Markov chain are completely determined by its one-step transition probability:

$$
p\left(x_{t-1}, x_{t}\right)=P\left(\frac{\left(X_{t}=x_{t}\right)}{\left(X_{t-1}=x_{t-1}\right)}\right)
$$

It is necessary to use Gibbs sampling to determine this conditional probability in the practical application of the Markov chain.

2.1.4. Gibbs Sampling. Gibbs sampling is the most famous MCMC sampling algorithm [31]. The process of one-step Gibbs sampling is given as follows:

(1) Extracting $x_{1}^{(t)}$ from $\pi\left(x_{1} /\left(x_{2}^{(t-1)}, \ldots, x_{n}^{(t-1)}\right)\right)$

(2) Extracting $x_{2}^{(t)}$ from $\pi\left(x_{2} /\left(x_{1}^{(t)}, x_{3}^{(t-1)}, \ldots, x_{n}^{(t-1)}\right)\right)$

(i) Extracting $x_{i}^{(t)}$ from $\pi\left(x_{i} /\left(x_{1}^{(t)}, x_{t-1}^{(t)}, x_{i-1}^{(t-1)}, \ldots\right.\right.$, $\left.\left.x_{n}^{(t-1)}\right)\right)$

$\ldots$

(n) Extracting $x_{n}^{(t)}$ from $\pi\left(x_{n} /\left(x_{1}^{(t)}, x_{2}^{(t)}, \ldots, x_{n-1}^{(t)}\right)\right)$

Its transition probability from $x$ to $x^{\prime}$ is as follows:

$p\left(x, x^{\prime}\right)=\pi\left(\frac{x_{1}}{x_{2}, \ldots, x_{n}}\right) \pi\left(\frac{x_{2}}{x_{1}^{\prime}, \ldots, x_{n}}\right), \ldots, \pi\left(\frac{x_{n}}{x_{1}^{\prime}, \ldots, x_{n-1}^{\prime}}\right)$.

Considering the Gibbs sampling as a multidimensional normal random vector, it is possible to set $X=\left(X_{1}, X_{2}\right)$ to obey the multivariate normal distribution as follows:

$$
\left(\frac{x_{1}^{(t)}}{x_{2}^{(t)}}\right)^{\sim} N\left(\left(\begin{array}{c}
\rho^{2 t-1} x_{2}^{(0)} \\
\rho^{2 t} x_{2}^{(0)}
\end{array}\right) \cdot\left(\begin{array}{cc}
1-\rho^{4 t-2} & 1-\rho^{4 t-1} \\
1-\rho^{4 t-1} & 1-\rho^{4 t}
\end{array}\right)\right) \text {. }
$$

Therefore, when $t \longrightarrow \infty$, the distribution of $\left(x_{1}^{(t)}, x_{2}^{(t)}\right)$ will converge to the target distribution.

\subsection{Bayesian Classifier and Bayesian Network}

2.2.1. Bayesian Classifier. The Bayesian classifier refers to a kind of the Bayesian network learning method [32]. Assume $U=\left\{A_{1}, A_{2}, \ldots, A_{n}, C\right\}$ is the $n$ attribute variables of the instance, it can be represented by the vector $x_{i}=\left(a_{1}, a_{2}, \ldots, a_{n}\right) . a_{i}$ is the value of $A_{i} . C$ is a class variable. $c$ is the value of $C$. The probability that $x_{i}$ belongs to the class $c_{j}$ is as follows:

$$
\begin{aligned}
p\left(\frac{c_{j}}{a_{1}, a_{2}, \ldots, a_{n}}\right) & =\frac{p\left(\left(a_{1}, a_{2}, \ldots, a_{n}\right) / c_{j}\right) p\left(c_{j}\right)}{p\left(a_{1}, a_{2}, \ldots, a_{n}\right)} \\
& =\alpha p\left(\frac{\left(a_{1}, a_{2}, \ldots, a_{n}\right)}{c_{j}}\right) p\left(c_{j}\right),
\end{aligned}
$$

where $\alpha$ is a regularization factor, $p\left(c_{j}\right)$ is the prior probability of $c_{j}$, and $p\left(c_{j} /\left(a_{1}, a_{2}, \ldots, a_{n}\right)\right)$ is the posterior probability of $c_{j}$. The prior probability is independent of the training data. According to the chain rule of probability, it can be expressed as follows:

$$
p\left(\frac{c_{j}}{a_{1}, a_{2}, \ldots, a_{n}}\right)=\alpha p\left(c_{j}\right) \prod_{i=1}^{n} p\left(a_{1}, a_{2}, \ldots, a_{i-1}, c_{j}\right) .
$$


Table 1: Descriptive statistics.

\begin{tabular}{|c|c|c|c|c|c|c|c|c|c|}
\hline & SP & Nasdaq & SSE & SZSE & HSI & FTSE & $\mathrm{CAC}$ & DAX & NIKKEI \\
\hline Mean & 0.220419 & 0.30801 & 0.119385 & 0.057389 & 0.10411 & 0.084644 & 0.143824 & 0.185196 & 0.271633 \\
\hline Median & 0.334225 & 0.504645 & 0.329614 & 0.248445 & 0.387111 & 0.051609 & 0.202949 & 0.249698 & 0.366232 \\
\hline Maximum & 4.39981 & 4.612395 & 7.701288 & 9.35729 & 6.904966 & 5.385454 & 6.90814 & 5.873106 & 7.37504 \\
\hline Minimum & -6.282521 & -5.916395 & -19.34068 & -20.0671 & -7.576548 & -5.242531 & -6.365694 & -6.209268 & -8.729473 \\
\hline Std. dev. & 1.29829 & 1.56905 & 2.79624 & 3.325197 & 1.968868 & 1.529423 & 1.917783 & 2.009933 & 2.309185 \\
\hline Skewness & -0.979637 & -0.780228 & -1.82048 & -1.668393 & -0.507765 & -0.237729 & -0.22026 & -0.361229 & -0.692988 \\
\hline Kurtosis & 7.232388 & 5.052937 & 12.70377 & 10.73481 & 4.283352 & 4.400866 & 4.286262 & 3.588966 & 5.342746 \\
\hline Jarque-Bera & 254.678 & 77.85544 & 1257.706 & 830.8407 & 31.35835 & 25.62351 & 21.64318 & 10.17253 & 86.75158 \\
\hline
\end{tabular}

TABLE 2: The simulation results of posterior parameters.

\begin{tabular}{|c|c|c|c|c|c|c|c|c|}
\hline node & Mean & Std. dev. & MC error & $2.50 \%$ & Median & $97.50 \%$ & Start & Sample \\
\hline$\mu_{s p}$ & 0.0529 & 0.2279 & 0.0117 & -0.4818 & 0.0849 & 0.4388 & 10000 & 100001 \\
\hline$\mu_{s h}$ & 1.012 & 0.4018 & 0.0192 & 0.18 & 1.022 & 1.774 & 10000 & 100001 \\
\hline$o$ & 10.47 & 3.714 & 0.1461 & 4.187 & 9.954 & 9.954 & 10000 & 100001 \\
\hline$\psi_{a a}$ & 0.8737 & 0.0972 & 0.0043 & 0.6238 & 0.8982 & 0.9896 & 10000 & 100001 \\
\hline$\psi_{a c}$ & 0.0423 & 0.0483 & 0.0023 & -0.0107 & 0.029 & 0.1804 & 10000 & 100001 \\
\hline$\psi_{c c}$ & 0.7671 & 0.1209 & 0.0063 & 0.5019 & 0.7853 & 0.9546 & 10000 & 100001 \\
\hline$\psi_{c a}$ & 0.5662 & 0.3106 & 0.0164 & 0.0647 & 0.5129 & 1.3140 & 10000 & 100001 \\
\hline$\sigma_{\xi_{a}}$ & 0.136 & 0.0454 & 0.0023 & 0.0744 & 0.128 & 0.2437 & 10000 & 100001 \\
\hline$\sigma_{\xi_{b}}^{a_{a}}$ & 0.1265 & 0.0539 & 0.0027 & 0.0622 & 0.1125 & 0.2679 & 10000 & 100001 \\
\hline
\end{tabular}

The key to use the Bayesian classification is how to calculate $p\left(a_{1}, a_{2}, \ldots, a_{i-1}, c_{j}\right)$. Different Bayesian classification models calculate it in different ways.

2.2.2. Bayesian Network. The Bayesian network is a directed acyclic graph (DAG). Each node corresponds to a conditional probability table as $P=P\left\{P\left(V_{i} / V_{1}, V_{2}, \ldots, V_{i-1}\right)\right.$, $\left.V_{i} \in V\right\}$.

The network structure $S$ is a DAG consisting of a set of node variables $V\left(V=\left\{V_{1}, V_{2}, \ldots, V_{n}\right\}\right)$ and a set of directed edges $L\left(L=\left\{\left(V_{i} V_{j} /\left(V_{i}, V_{j}\right)\right), t \in n V\right\}\right)$. The network structure is expressed as follows: $S=(V, L)$.

\section{Empirical Analysis}

3.1. Data and Preprocessing. In this section, the DGC-t-MSV model proposed in Section 2.1 is fitted to the historical stock market data. We choose the most popular index, including S\&P, Nasdaq, SSE, SZSE, HSI, FTSE, CAC, DAX, and Nikkei. These are representative stock indices in America, Europe, and Asia. We collected the daily price from January 2, 2013, to May 15, 2018. Then, we calculate 282 weekly data and standardize them. They can reflect the cross-market volatility between the world's major economies. The S\&P and Nasdaq are both major markets in America. The British FTSE, French CAC, and German DAX are known as the three major European stock indices. The SSE and SZSE are the emerging markets which are growing fast. The Nikkei and HSI are both important and developed markets in Asia. The weekly data are used to eliminate the week effects and the different time zones of the global market. The descriptive statistics is shown in Table 1.
3.2. Parameter Estimation. In this paper, Bayesian simulation is performed by the WinBUGs package. Taking S\&P index and SSE index as examples, we abandon the first 10,000 iterations as the so-called "burn-in." Then, we simulate the last 100,000 iterations to obtain the posterior parameters, as shown in Table 2.

In Table 2, $\psi_{a c}$ represents the volatility spillover from the SSE index to the S\&P index. The mean value of $\psi_{a c}$ is less than 0.1 , which means the spillover from the SSE to the S\&P index is not significant. $\psi_{c a}$ has a mean value of 0.5662 , which is greater than 0.1 . So the spillover from the S\&P to the SSE is significant. The volatility level parameter $\mu_{s p}$ of the $\mathrm{S} \& \mathrm{P}$ is 0.0529 . And $\mu_{s h}$ of the SSE index is 1.012. Obviously, the absolute value of the volatility level $\mu_{s h}$ is greater than $\mu_{s p}$, reflecting that the risk of the Shanghai stock market is higher than that of the S\&P market. The volatility persistence parameter $\psi_{a a}$ of the S\&P index is 0.8737 , and $\psi_{c c}$ of the SSE index is 0.7671 . The S\&P index volatility persistence is stronger than the SSE index.

Figure 1 shows that $\mu_{s p}$ and $\mu_{s h}$ are convergent. Each parameter has a MCMC error of less than $5 \%$ of the sample standard deviation (SD). Other parameter results are convergent too. We do not show them in the text due to space limitations. It can be seen from Figure 2 that the dynamic correlation coefficient $\rho$ is higher. All estimated results of volatility spillover parameters are shown in Table 3 . We use NA, SP, SH, SZ, HK, JP, UK, FA, and GE to represent Nasdaq, S\&P, SSE, SZSE, HSI, Nikkei, FTSE, CAC, and DAX indices.

3.3. Bayesian Network. The volatility spillover relationship is a ring diagram. It does not allow a ring diagram in the Bayesian network. In order to facilitate the research, it is 


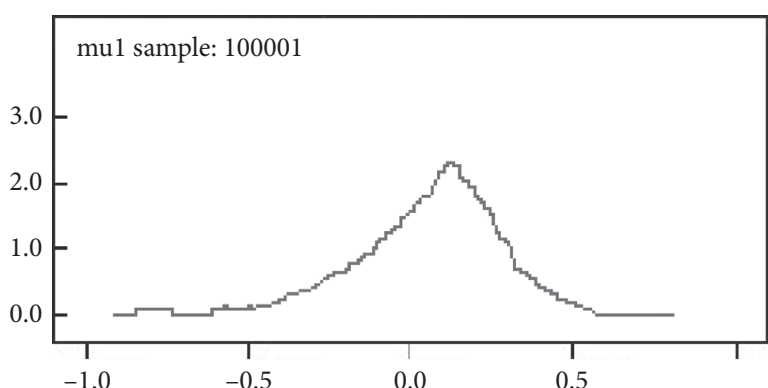

(a)

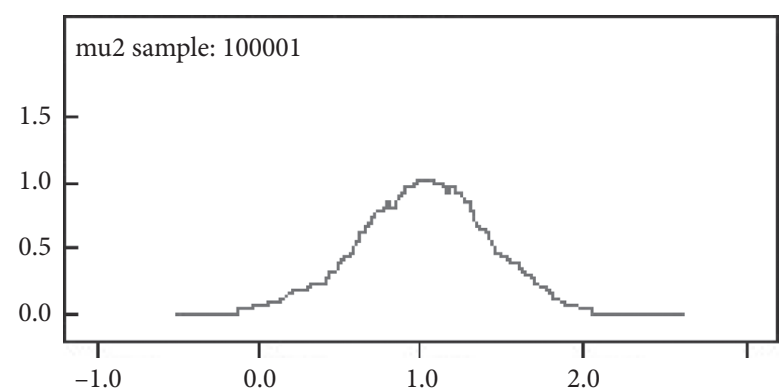

(b)

FIgURE 1: $\mu_{s p}$ and $\mu_{\text {sh }}$ posterior distribution density.

TABLE 3: Volatility spillover parameter estimation result.

\begin{tabular}{lccccccrrr}
\hline & NA & SP & SH & SZ & HK & JP & UK & FA & GE \\
\hline NA & - & 0.2393 & 0.6552 & 0.6354 & -0.003 & 0.322 & -0.031 & 0.2401 & 0.2156 \\
SP & 0.1 & - & 0.5662 & 0.3968 & 0.0087 & 0.1833 & -0.163 & 0.1108 & 0.1089 \\
SH & 0.0397 & 0.0423 & - & 0.2786 & 0.0557 & 0.0337 & 0.1086 & 0.1534 & 0.1137 \\
SZ & 0.0434 & 0.0594 & 0.0872 & - & 0.0711 & 0.0287 & 0.1608 & 0.1077 & 0.129 \\
HK & 0.4577 & 0.0087 & 0.6744 & 0.6684 & - & 0.6907 & 0.401 & 0.3567 & 0.3122 \\
JP & 0.0132 & 0.0168 & 0.3417 & 0.3751 & 0.0257 & - & 0.1807 & 0.1608 & 0.0599 \\
UK & 0.2809 & 0.3848 & 0.2952 & 0.1784 & 0.0586 & 0.0365 & - & 0.0967 & 0.152 \\
FA & 0.0400 & 0.0360 & 0.195 & 0.3979 & 0.0783 & 0.1009 & 0.2286 & - & 0.1771 \\
GE & 0.0641 & 0.0729 & 0.2627 & 0.2722 & 0.1136 & 0.207 & 0.217 & 0.2232 \\
\hline
\end{tabular}

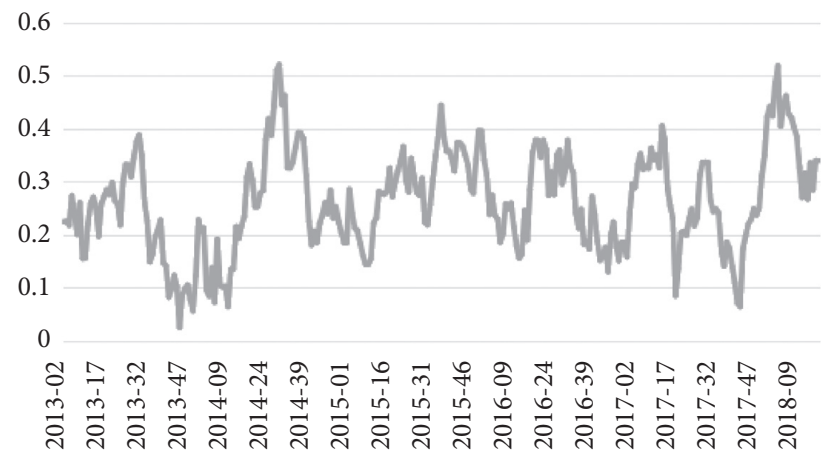

Figure 2: Correlation $\rho$ between the S\&P index and Shanghai Composite Index.

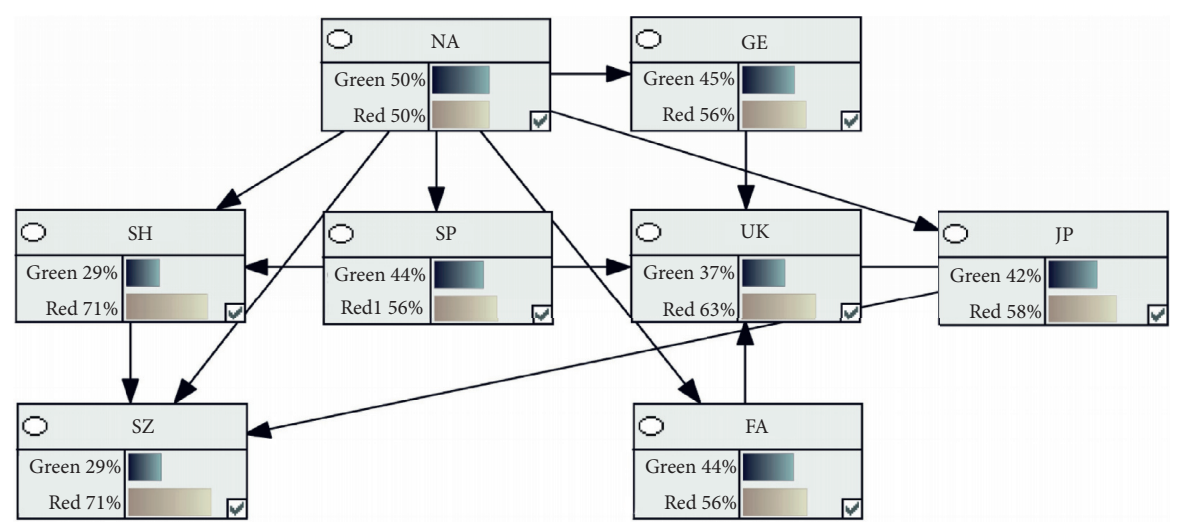

Figure 3: BN initial state diagram (NA). 


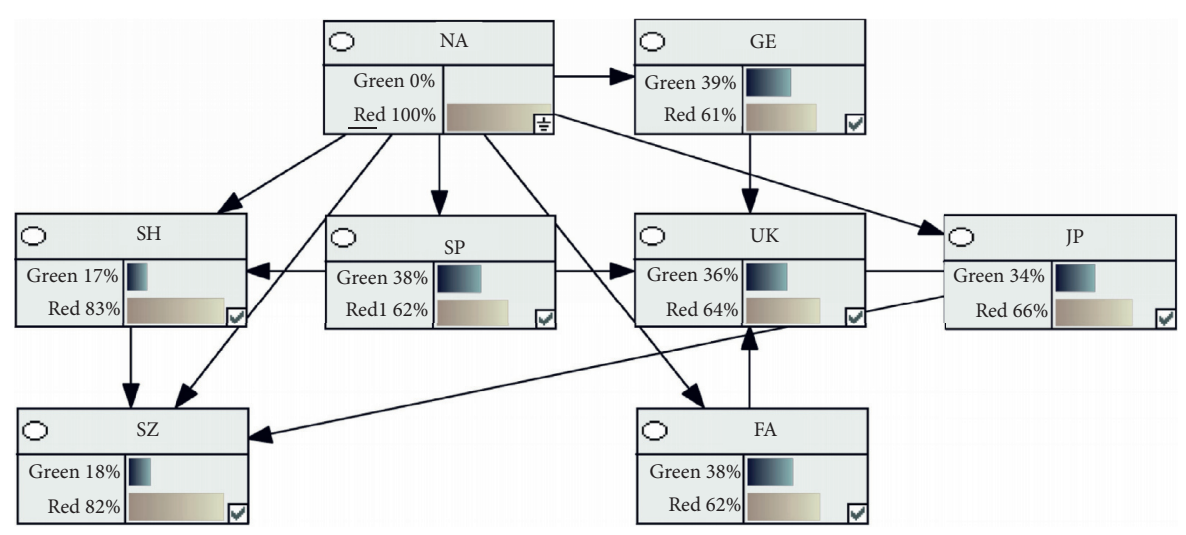

Figure 4: BN final state diagram (NA).

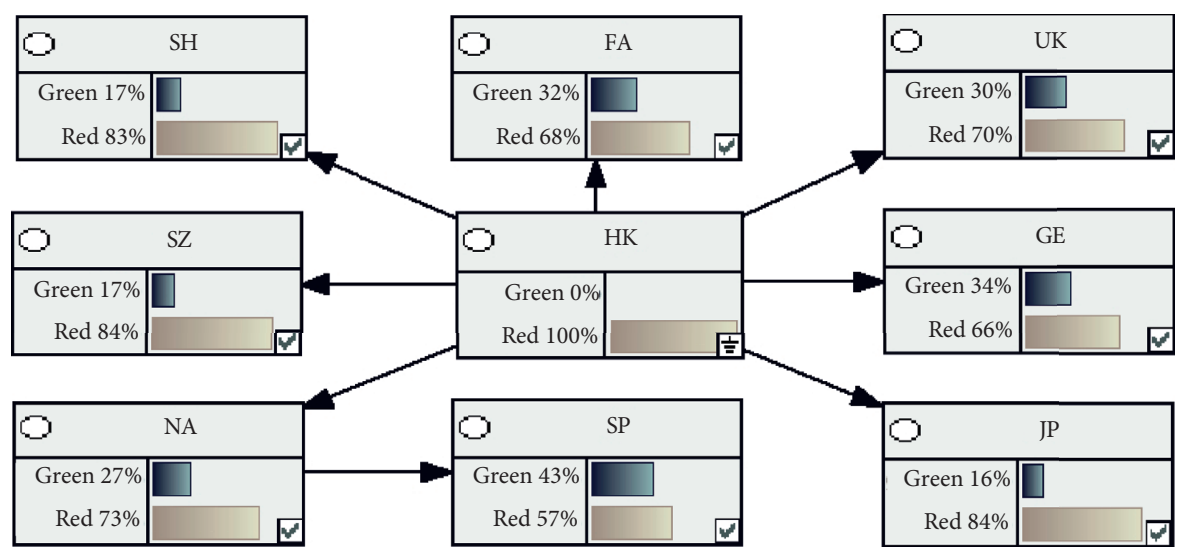

Figure 5: BN final state diagram (HK).

necessary to extract the directed acyclic graph from a certain node. This paper uses GeNie software to establish the Bayesian network. GeNie software is one of the commonly used software for Bayesian network simulation. We assume that the initial probability of herd infection between markets is consistent. Based on the significant volatility spillover parameters in Table 2, the relationship between the Nasdaq market and other markets is extracted, as shown in Figure 3.

In ordinary, the infection probability of herd behavior between each market is shown in Figure 3. In Figure 4, we test the spillover effect caused by the Nasdaq market when it has a terrible crisis. We assume that the HK stock market is totally emerging as a herd behavior, as shown in Figure 5. In Figure 5, it can be found that the herd behavior in Hong Kong will cause a large volatility spillover in Asia, generally higher than $80 \%$. The market in Europe and US is not sensitive to the crisis of Hong Kong. It shows that the Asian investors are highly concerned about Hong Kong.

\section{Conclusion}

This paper analyzes the volatility spillover using the DGC-tMSV model and Bayesian network. The above calculation results and the conclusions of the analysis can reflect the current situation of international stock markets, indicating that the method used in this paper is effective.
(1) Analysis of volatility spillover shows that there is an extensive linkage between international markets. Most developed stock markets will quickly respond to abnormal impact in major global stock markets, such as the Hong Kong stock market. The Bayesian network simulation proves that the Hong Kong stock market has a wide range of influence. The Nasdaq and S\&P indices reflect the high global influence of the US stock market. Therefore, we must pay more attention to the contagious and influential stock markets, such as the Nasdaq and S\&P. This result will help investors to understand the volatility correlation between stock markets and explain some impact factors such as crossmarket herd behavior. (2) By establishing a Bayesian network, it can be found that the stock market is more sensitive in one economic region. This paper uses weekly data to avoid the effects of the week and the time difference. It can be found that, in Europe, the British FTSE Index, the French CAC40 Index, and the German DAX30 Index are more closely related. In the Asian market, the Shanghai SSE Index, the Shenzhen SZSE Index, and the Hong Kong HSI Index are more closely related. This is an obvious phenomenon of regional economics. In general, the spread of herd behavior is related to the regional division. This result shows that investors should pay more attention to the crisis in the surrounding markets. (3) Using the MSV model and the Bayesian network, it can be found that the Chinese stock 
market is more closely linked to the foreign stock market, especially the US market. This result can reflect the trading relationship between China and US.

The research in this paper shows that stock investors should avoid some highly correlated markets when they allocate diversified stock assets. They can lower the risk by choosing the market with lower correlation. The DGC-tMSV model used in this paper can simulate the impact of multiple random factors on the market. The Bayesian network can clearly reflect the relationship between multiple markets. Further research will use the self-learning method to learn these random factors in the Bayesian network.

\section{Data Availability}

The data used to support the findings of this study are included within the article.

\section{Conflicts of Interest}

The authors declare there are no conflicts of interest regarding the publication of this paper.

\section{Authors' Contributions}

All authors contributed equally to this study.

\section{Acknowledgments}

This work was supported by the Major Projects of Social Sciences in Anhui Universities (SK2020ZD006) and the General Project of Anhui Natural Science Foundation (1908085MG232).

\section{References}

[1] T. Bollerslev, "Generalized autoregressive conditional heteroskedasticity," Journal of Econometrics, vol. 31, no. 3, pp. 307-327, 1986.

[2] S. Kim, N. Shepherd, and S. Chib, "Stochastic volatility: likelihood inference and comparison with arch models," Review of Economic Studies, vol. 65, no. 3, pp. 361-393, 1998.

[3] M. V. Kulikova and D. R. Taylor, "Stochastic volatility models for exchange rates and their estimation using quasi-maximum-likelihood methods: an application to the South African rand," Journal of Applied Statistics, vol. 40, no. 3, pp. 495-507, 2013.

[4] W. Surapaitoolkorn, "Variable dimension via stochastic volatility model using FX rates," Journal of Applied Statistics, vol. 40, no. 10, pp. 2110-2128, 2013.

[5] H. Ghosh, B. Gurung, and Prajneshu, "Kalman filter-based modelling and forecasting of stochastic volatility with threshold," Journal of Applied Statistics, vol. 42, no. 3, pp. 492-507, 2015.

[6] D. B. Nugroho and T. Morimoto, "Box-Cox realized asymmetric stochastic volatility models with generalized Student'st-error distributions," Journal of Applied Statistics, vol. 43, no. 10, pp. 1906-1927, 2016.

[7] S. Chib, F. Nardari, and N. Shephard, "Analysis of high dimensional multivariate stochastic volatility models," Journal of Econometrics, vol. 134, no. 2, pp. 341-371, 2006.
[8] Y. Omori, S. Chib, N. Shephard, and J. Nakajima, "Stochastic volatility with leverage: fast and efficient likelihood inference," Journal of Econometrics, vol. 140, no. 2, pp. 425-449, 2007.

[9] M. Zhongxian, D. McLeish, A. W. Kolkiewicz, and T. S. Wirjanto, "Comparison of asymmetric stochastic volatility models under different correlation structures," Journal of Applied Statistics, vol. 44, no. 8, pp. 1350-1368, 2017.

[10] K. Jacobs and X. Li, "Modeling the dynamics of credit spreads with stochastic volatility," Management Science, vol. 54, no. 6, pp. 1176-1188, 2008.

[11] C.-C. Nieh, C.-H. Yang, and Y.-S. Kao, "Who has more influence on asian stock markets around the subprime mortgage crisis - the US or China?" Applied Economics Letters, vol. 19, no. 4, pp. 329-335, 2012.

[12] T. Chen, "Do investors herd in global stock markets?" Journal of Behavioral Finance, vol. 14, no. 3, pp. 230-239, 2013.

[13] T. T.-L. Chong, X. Liu, and C. Zhu, "What explains herd behavior in the Chinese stock market?" Journal of Behavioral Finance, vol. 18, no. 4, pp. 448-456, 2017.

[14] W.-R. Yang and Y.-L. Chen, "The response of dynamic herd behavior to domestic and u. s. market factors: evidence from the greater China stock markets," Emerging Markets Finance and Trade, vol. 51, no. sup1, pp. S18-S41, 2015.

[15] F. Economou, C. Hassapis, and N. Philippas, "Investors' fear and herding in the stock market," Applied Economics, vol. 50, no. 34-35, pp. 3654-3663, 2018.

[16] K. Lee, "Herd behavior of the overall market: evidence based on the cross-sectional comovement of returns," The North American Journal of Economics and Finance, vol. 42, pp. 266-284, 2017.

[17] W. Lee, Y. H. Choi, C. Kim, and J. Y. Ahn, "A case study for intercontinental comparison of herd behavior in global stock markets," Communications for Statistical Applications and Methods, vol. 25, no. 2, pp. 185-197, 2018.

[18] E. Baumöhl, E. Kočenda, S. Lyócsa, and T. Výrost, "Networks of volatility spillovers among stock markets," Physica A: Statistical Mechanics and Its Applications, vol. 490, pp. 1555-1574, 2018.

[19] I. Younis, C. Longsheng, M. F. Basheer, and A. S. Joyo, "Stock market comovements among asian emerging economies: a wavelet-based approach," PLoS ONE, vol. 15, no. 10, pp. 1-23, 2020.

[20] C. Aloui, B. Hkiri, and D. K. Nguyen, "Real growth comovements and business cycle synchronization in the gcc countries: evidence from time-frequency analysis," Economic Modelling, vol. 52, pp. 322-331, 2016.

[21] S. Zhao, X. Chen, and J. Zhang, “The systemic risk of China's stock market during the crashes in 2008 and 2015," Physica A: Statistical Mechanics and Its Applications, vol. 520, pp. 161177, 2019.

[22] M. A. Cengiz, E. Dünder, and T. Şenel, "Energy performance evaluation of oecd countries using bayesian stochastic frontier analysis and bayesian network classifiers," Journal of Applied Statistics, vol. 45, no. 1, pp. 17-25, 2018.

[23] G. Feng and X. Zhang, "Productivity and efficiency at large and community banks in the us: a bayesian true random effects stochastic distance frontier analysis," Journal of Banking \& Finance, vol. 36, no. 7, pp. 1883-1895, 2012.

[24] W. Long, L. Guan, J. Shen, L. Song, and L. Cui, "A complex network for studying the transmission mechanisms in stock market," Physica A: Statistical Mechanics and Its Applications, vol. 484, pp. 345-357, 2017. 
[25] J. L. Ticknor, "A bayesian regularized artificial neural network for stock market forecasting," Expert Systems with Applications, vol. 40, no. 14, pp. 5501-5506, 2013.

[26] L. Wang, Z. Wang, S. Zhao, and S. Tan, "Stock market trend prediction using dynamical bayesian factor graph," Expert Systems with Applications, vol. 42, no. 15-16, pp. 6267-6275, 2015.

[27] R. Oh, D. W. Shin, and M.-S. Oh, "Bayesian analysis of financial volatilities addressing long-memory, conditional heteroscedasticity and skewed error distribution," Communications for Statistical Applications and Methods, vol. 24, no. 5, pp. 507-518, 2017.

[28] L. S. Malagrino, N. T. Roman, and A. M. Monteiro, "Forecasting stock market index daily direction: a bayesian network approach," Expert Systems with Applications, vol. 105, pp. 11-22, 2018.

[29] J. Yu and R. Meyer, "Multivariate stochastic volatility models: bayesian estimation and model comparison," Econometric Reviews, vol. 25, no. 2-3, pp. 361-384, 2006.

[30] J. B. Liu, J. Zhao, and Z. Q. Cai, "On the generalized adjacency, Laplacian and signless Laplacian spectra of the weighted edge corona networks," Physica A-Statistical Mechanics and Its Applications, vol. 540, 2020.

[31] Z. Zhu and J.-B. Liu, "The normalized Laplacian, degreeKirchhoff index and the spanning tree numbers of generalized phenylenes," Discrete Applied Mathematics, vol. 254, pp. 256-267, 2019.

[32] J. B. Liu and S. N. Daoud, "Number of spanning trees in the sequence of some graphs," Complexity, vol. 2019, Article ID 4271783, 2019. 